\title{
Novel White Matter Tract Integrity Metrics Sensitive to Alzheimer Disease Progression
}

\author{
E. Fieremans, A. Benitez, J.H. Jensen, M.F. Falangola, A. Tabesh, R.L. Deardorff, M.V.S. Spampinato, J.S. Babb, D.S. Novikov,
} S.H. Ferris, and J.A. Helpern

\begin{abstract}
BACKGROUND AND PURPOSE: Along with cortical abnormalities, white matter microstructural changes such as axonal loss and myelin breakdown are implicated in the pathogenesis of Alzheimer disease. Recently, a white matter model was introduced that relates nonGaussian diffusional kurtosis imaging metrics to characteristics of white matter tract integrity, including the axonal water fraction, the intra-axonal diffusivity, and the extra-axonal axial and radial diffusivities.
\end{abstract}

MATERIALS AND METHODS: This study reports these white matter tract integrity metrics in subjects with amnestic mild cognitive impairment $(n=12)$, Alzheimer disease $(n=14)$, and age-matched healthy controls $(n=15)$ in an effort to investigate their sensitivity, diagnostic accuracy, and associations with white matter changes through the course of Alzheimer disease.

RESULTS: With tract-based spatial statistics and region-of-interest analyses, increased diffusivity in the extra-axonal space (extra-axonal axial and radial diffusivities) in several white matter tracts sensitively and accurately discriminated healthy controls from those with amnestic mild cognitive impairment (area under the receiver operating characteristic curve $=0.82-0.95$ ), while widespread decreased axonal water fraction discriminated amnestic mild cognitive impairment from Alzheimer disease (area under the receiver operating characteristic curve $=0.84$ ). Additionally, these white matter tract integrity metrics in the body of the corpus callosum were strongly correlated with processing speed in amnestic mild cognitive impairment $(r=|0.80-0.82|, P<.001)$.

CONCLUSIONS: These findings have implications for the course and spatial progression of white matter degeneration in Alzheimer disease, suggest the mechanisms by which these changes occur, and demonstrate the viability of these white matter tract integrity metrics as potential neuroimaging biomarkers of the earliest stages of Alzheimer disease and disease progression.

ABBREVIATIONS: $A D=$ Alzheimer disease; $\mathrm{AMCl}=$ amnestic mild cognitive impairment; $\mathrm{AWF}=$ axonal water fraction; $\mathrm{CPS}=$ composite processing speed; $\mathrm{DKI}=\mathrm{diffusional}$ kurtosis imaging; FA = fractional anisotropy; NC = healthy controls; TBSS = tract-based spatial statistics; WMTI = white matter tract integrity

A lzheimer disease $(\mathrm{AD})$ is a disorder with a pathologic cascade that long precedes its clinical manifestations, ${ }^{1}$ motivating researchers to develop biomarkers of early pathologic changes on

Received November 30, 2012; accepted after revision January 23.

From the Department of Radiology (E.F., J.S.B., D.S.N.), Center for Biomedical Imaging, and Department of Psychiatry (S.H.F.) and Alzheimer's Disease Center, New York University School of Medicine, New York, New York; and Department of Radiology and Radiological Science (A.B., J.H.J., M.F.F., A.T., R.L.D., M.V.S.S., J.A.H.), Center for Biomedical Imaging (A.B., J.H.J., M.F.F., A.T., R.L.D., M.V.S.S., J.A.H.), and Department of Neurosciences (M.F.F., J.A.H.), Medical University of South Carolina, Charleston, South Carolina.

E. Fieremans and A. Benitez contributed equally to the work.

This work was supported by the Alzheimer Drug Discovery Foundation, the Litwin Foundation, and the NIH (R01AG027852).

Preliminary analyses of some of these data previously presented at: 20th Annual Meeting of the International Society for Magnetic Resonance in Imaging, May 5-11, 2012; Melbourne, Australia; and the Alzheimer's Association International Conference, July 14-19, 2012; Vancouver, British Columbia.

Please address correspondence to Els Fieremans, PhD, Department of Radiology, New York University School of Medicine, 660 First Ave, 4th floor, New York, NY 10016; e-mail: Els.Fieremans@nyumc.org which therapies could more effectively focus. ${ }^{2}$ Along with wellcharacterized cortical abnormalities, ${ }^{3}$ postmortem studies have also provided evidence of pathologic changes in WM occurring early in the course of $\mathrm{AD}$, including decreased myelin and axonal attenuation, ${ }^{4-7}$ loss of oligodendrocytes, ${ }^{8}$ and activation of glial cells. $^{7}$ Diffusion MRI allows the study of WM pathology in AD because it measures the $\mu \mathrm{m}$-scale displacement of water molecules to index microstructural (ie, dendritic, axonal, and myelin) loss, which, in a recent meta-analysis, has been shown to better differentiate normal aging from mild cognitive impairment and AD than hippocampal atrophy. ${ }^{9}$ Additionally, a comprehensive review of diffusion MRI studies of the largest cerebral WM tract, the corpus callosum, illustrated the distinct pathophysiologies underlying $\mathrm{WM}$ microstructural changes in $\mathrm{AD}^{10}$; the anterior

\footnotetext{
- Indicates open access to non-subscribers at www.ajnr.org

$\equiv$ Indicates article with supplemental on-line appendix

http://dx.doi.org/10.3174/ajnr.A3553
} 
Table 1: Demographic and medical history descriptive statistics for healthy controls, patients with amnestic $\mathrm{MCl}$, and subjects with AD $(N=41)$

\begin{tabular}{|c|c|c|c|c|c|}
\hline & $\begin{array}{c}\text { NC }(n=15) \\
n(\%)\end{array}$ & $\begin{array}{c}\mathrm{aMCl}(n=12) \\
\mathrm{n}(\%)\end{array}$ & $\begin{array}{c}\mathrm{AD}(n=14) \\
n(\%)\end{array}$ & $\chi^{2} / F$-test & $P$ Value \\
\hline \multicolumn{6}{|l|}{ Demographics } \\
\hline Age (mean) (yr) & $77.54 \pm 4.01$ & $79.05 \pm 7.23$ & $78.30 \pm 9.55$ & 0.15 & .87 \\
\hline Female & $10(66)$ & $6(50)$ & $8(57)$ & 0.78 & .68 \\
\hline Education (mean $\pm S D$ ) & $16.14 \pm 2.51$ & $15.08 \pm 3.66$ & $15.14 \pm 2.83$ & 0.54 & .59 \\
\hline Right-handed & $13(87)$ & $11(92)$ & $13(93)$ & 1.94 & .75 \\
\hline White race & $14(93)$ & $10(83)$ & $12(86)$ & 4.46 & .62 \\
\hline \multicolumn{6}{|l|}{ Medical history } \\
\hline Hypertension & $8(53)$ & $8(67)$ & $4(29)$ & 3.95 & .14 \\
\hline Hypercholesterolemia & $12(80)$ & $9(75)$ & $6(43)$ & 3.09 & .21 \\
\hline Diabetes & $2(13)$ & $1(8)$ & $1(7)$ & 0.35 & .84 \\
\hline Thyroid disease & $3(20)$ & 2 (17) & $1(7)$ & 1.01 & .60 \\
\hline Cardiac arrest & $2(13)$ & $3(25)$ & $1(7)$ & 2.01 & .37 \\
\hline Cardiac bypass & $1(7)$ & $1(8)$ & $0(0)$ & 1.13 & .57 \\
\hline Stroke or TIA & $1(7)$ & $1(8)$ & $1(7)$ & 0.03 & .99 \\
\hline Depression within the past 2 years & $4(27)$ & $1(8)$ & $4(29)$ & 1.85 & .40 \\
\hline
\end{tabular}

Note:-TIA indicates transient ischemic attack

part of the corpus callosum (ie, the genu) appears to degenerate due to myelin breakdown, ${ }^{11,12}$ while decline in the posterior part (ie, the splenium) is attributed to Wallerian degeneration ${ }^{13}$ secondary to distal gray matter $\mathrm{AD}$ pathology in the temporoparietal areas to which the posterior callosum projects. ${ }^{6}$

To date, most diffusion MR imaging studies of WM pathology in $\mathrm{AD}$ and mild cognitive impairment have used DTI. ${ }^{14}$ Here, we use diffusional kurtosis imaging (DKI), a clinically feasible extension of DTI that also examines the non-Gaussian diffusion effects of water known to occur in the brain. ${ }^{15,16}$ While both DTI-derived and DKI-derived metrics may be altered in $\mathrm{AD},{ }^{17}$ they lack microstructural and pathologic specificity. Indeed, empirical diffusion measures only provide indirect measurements of microstructure, making their physical meaning in terms of microscopic tissue parameters uncertain. To overcome this limitation, we used a WM model that was recently proposed to relate DKI-derived metrics directly to WM microstructure ${ }^{18}$ by introducing WM tract integrity (WMTI) metrics, namely axonal water fraction (AWF), intra-axonal diffusivity $\left(D_{\text {axon }}\right)$, and extra-axonal axial and radial diffusivities $\left(D_{e, \|}\right.$ and $\left.D_{e, \perp}\right)$. These white matter tract integrity (WMTI) metrics are, by design, more specific to the underlying mechanisms of WM alterations than conventional diffusion measures.

In this study, we sought to demonstrate the utility of WMTI metrics in further clarifying the WM changes that occur as $\mathrm{AD}$ progresses. We also investigated the extent to which these metrics correlate with processing speed, a cognitive function known to decline due to aging, disease, and WM compromise. Our findings contribute to the growing literature on WM involvement in AD and offer novel candidate biomarkers of $\mathrm{AD}$ progression.

\section{MATERIALS AND METHODS \\ Subjects and Procedures}

Subjects were recruited from the NYU Alzheimer Disease Center. All subjects provided written informed consent before participating in this study, which was approved by the institutional review board of the NYU School of Medicine. Per study procedures, all subjects underwent full neurologic and psychiatric evaluations, a neuropsychological test battery, and an MR imaging brain scan.
All diagnoses followed research criteria: healthy controls (NC) $(n=15)$ had no evidence of dementia or mild cognitive impairment and had a Global Deterioration Scale score ${ }^{19}$ of 1-2; subjects with amnestic mild cognitive impairment (aMCI) $(n=12)$ were defined as having a self- and/or informant-reported memory loss, memory impairment based on performance that was at least $1 \mathrm{SD}$ below the normative mean for age on Logical Memory-II of the Wechsler Memory Scale, ${ }^{20}$ a Global Deterioration Scale score $=3$, and no dementia. Subjects with $\mathrm{AD}(n=14)$ were given a diagnosis based on the Diagnostic and Statistical Manual of Mental Disorders- $I V^{21}$ and the National Institute of Neurological Disorders and Stroke/Alzheimer's Disease and Related Disorders Association Criteria for probable $\mathrm{AD}^{22}$ by a Global Deterioration Scale score $=4-5$ and were not deemed to have any medical, neurologic, or psychiatric conditions that could otherwise account for the dementia. The groups significantly differed in their Mini-Mental State Examination $^{23}$ scores $[F(2,38)=18.60, P<.001]$, in which NC $(29.33 \pm 0.72)$ and subjects with aMCI $(27.75 \pm 1.71)$ had significantly higher scores $(P<.001)$ than subjects with $\mathrm{AD}(21.64 \pm 5.80)$. Table 1 summarizes the subject demographic information and selfreported medical history.

\section{Processing Speed}

The 2 tests used in this study are classified under the cognitive domain of processing speed in the National Institute on AgingAlzheimer's Disease Research Center designated Uniform Dataset neuropsychological battery. ${ }^{24}$ In the Digit Symbol Substitution test, subjects are asked to rapidly copy symbols that correspond to a set of integers according to a provided key, in which a higher number of correctly copied symbols in 90 seconds indicates faster processing speed. In the Trail-Making Test-A, subjects are asked to rapidly connect 25 consecutively numbered circles. The test score is the time (in seconds) in which the participant completes the task; a lower completion time indicates faster processing speed. To facilitate the interpretation and analyses of these measures, we converted the raw score for each test to a $z$ score by using the age, sex, and education normative data from the Uniform Dataset, ${ }^{25}$ and both $z$ scores were averaged to create a composite processing speed (CPS) $z$ score. As expected, the 3 groups 
differed on CPS $[F(2,38)=25.07, P<.001]$, with the subjects with $\mathrm{AD}(-2.47 \pm 1.62)$ scoring significantly lower $(P<.001)$ than the $\mathrm{NC}(0.41 \pm 0.55)$ and subjects with aMCI $(0.15 \pm$ $1.19)$.

\section{MR Imaging Acquisition}

MR imaging experiments were conducted on a 3T Trio MR imaging system (Siemens Medical Solutions, Erlangen, Germany) by using a 12-channel head coil. The MR imaging protocol, including DKI, is discussed in the On-line Appendix, Section 1.

\section{WMTI Characterization with DKI}

DKI provides both the diffusion and kurtosis tensors, from which standard diffusivity metrics (ie, mean diffusivity $[\mathrm{MD}]$, axial diffusivity $\left[D_{\|}\right]$, radial diffusivity $\left.\left.\left[\mathrm{D}_{\perp}\right]\right),\left[D_{] \text {perp }]}\right]\right)$, fractional anisotropy (FA), and kurtosis metrics (ie, mean kurtosis [MK], axial kurtosis $\left[K_{\|}\right]$, and radial kurtosis $\left.\left[K_{\perp}\right]\right)$ can be derived. ${ }^{15,16}$ In addition, we recently introduced WM tract integrity metrics by defining a WM model that relates these DKI-derived metrics directly to WM microstructure. ${ }^{18,26}$ In this model, we characterized WM microstructure that consists of aligned fiber bundles by assuming that the tissue comprises cylindrical axons, each surrounded by a myelin sheath. The relative volume of water within the collective intra-axonal space represents the axonal water fraction. The remainder of the WM is modeled as the extra-axonal space. Through the mathematic derivation described in Fieremans et al, ${ }^{18}$ the WM model metrics (ie, AWF and the individual compartmental diffusion tensors for the intra-axonal space and extra-axonal space) can be calculated from the diffusion and kurtosis tensors. In this study, we focused on the following WMTI metrics and their proposed interpretation ${ }^{27,28}$ :

-AWF (ie, axonal water fraction, a marker of axonal density)

- $D_{\text {axon }}$ (ie, the intrinsic diffusivity inside the axons, a marker of axonal injury)

$\bullet D_{\mathrm{e}, \|}$ and $D_{\mathrm{e}, \perp}$ (the axial and radial diffusivity in the EAS, markers of isotropic changes in extra-axonal diffusion [eg, due to gliosis, loss of oligodendrocytes, or extracellular inflammation]). In addition, $D_{\mathrm{e}, \perp}$ is a marker for changes in extra-axonal diffusion transverse to the fibers (eg, due to myelin breakdown).

\section{Image Processing and Analysis}

Image processing and analysis are discussed in the On-line Appendix, Section 2. Both voxelwise analysis by using the standard procedure of tract-based spatial statistics (TBSS) ${ }^{29,30}$ and region-of-interest analysis of the corpus callosum and the skeleton were performed by using the FMRIB Software Library (FSL, http://www.fmrib.ox.ac.uk/fsl).

\section{Statistical Analyses}

Statistical analyses are discussed in the On-line Appendix, Section 3. Because this is the first demonstration of the value of the recently proposed WMTI metrics in detecting in vivo alterations in $\mathrm{WM}$ architecture in the course of $\mathrm{AD}$, we adopted an exploratory approach of performing both a voxelwise analysis by using the standard procedure of tract-based spatial statistics ${ }^{29,30}$ and a ROI analysis of the corpus callosum to test differences between groups. Area under the receiver operating characteristic curve and linear discriminant analyses were conducted to assess the diagnostic
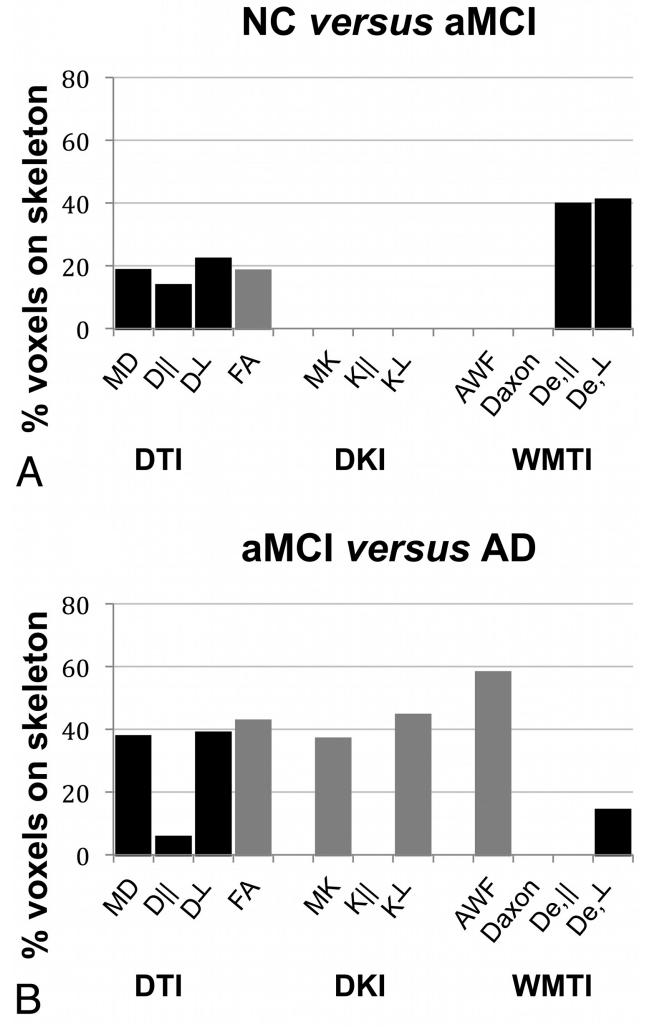

NC versus $A D$

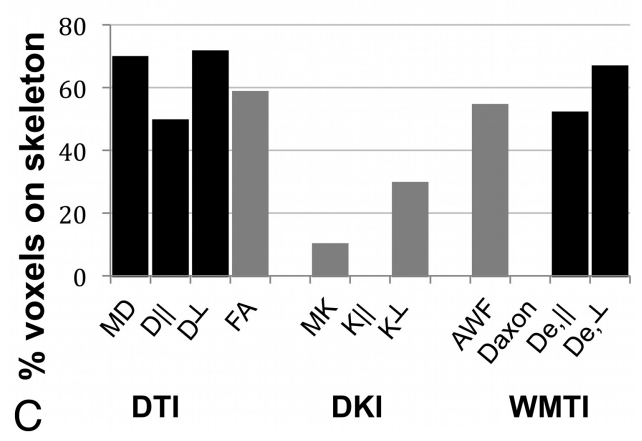

FIG 1. Bar graphs showing the percentage of significantly different voxels on the skeleton for each standard diffusivity, kurtosis, and WMTI metric and group comparison $(A, N C$ versus aMCl; $B, a \mathrm{MCl}$ versus $A D ; C, N C$ versus $A D$ ) using TBSS. Black bars indicate increased; gray bars, decreased.

utility of each regional metric in differentiating each group. Last, Spearman correlations of the age-residualized WMTI metrics of the ROIs and CPS score were conducted.

\section{RESULTS}

\section{WMTI Metrics Sensitively Detect Group Differences}

With TBSS, the percentage of significantly different voxels (onesided $P<.05$, corrected for multiple comparisons) for each metric and group comparison is shown in Fig 1 to demonstrate the overall sensitivity of each diffusion measure to discriminate among the groups. Figure $1 A$ shows the results for the NC versus aMCI groups: All standard diffusivity metrics were found to be significantly different between the $\mathrm{NC}$ and aMCI groups, with the diffusion coefficients $\left(\mathrm{MD}, D_{\|}\right.$, and $\left.D_{\perp}\right)$ being increased and the 
FA decreased. Among all diffusion metrics examined, the metrics with the highest number of voxels that were significantly different between the NC and aMCI groups were the WMTI extra-axonal diffusivities, $D_{\mathrm{e} \|}$ and $D_{\mathrm{e} \perp}$, both significantly increased in $37 \%$ and $38 \%$ of the voxels on the skeleton, respectively. Figure $1 B$ shows the results for the aMCI versus AD groups: All standard diffusivity metrics were found to be significantly different, with the diffusion coefficients being increased and FA decreased. The kurtosis metrics $\mathrm{MK}$ and $K_{\perp}$ were also found to be significantly decreased. The AWF had the highest number of voxels (ie, 55\%) that were significantly decreased. $D_{\mathrm{e}, \perp}$ was also significantly increased, but in fewer voxels. Figure $1 C$ shows the results for the $\mathrm{NC}$ versus $\mathrm{AD}$ groups, where all metrics except $K_{\|}$and $D_{\text {axon }}$ were found to be significantly different, with the highest sensitivities for the metrics $\operatorname{MD}(67 \%), D_{\perp}(69 \%)$, and $D_{e, \perp}(64 \%)$.

Figure 2 shows the spatial distribution of the TBSS analysis for the WMTI metrics that were found to be the most sensitive in differentiating $\mathrm{NC}$ from patients with aMCI, and patients with aMCI from those with $\mathrm{AD}$ (ie, $D_{e, \perp}, D_{e, \|}$, and AWF). When we compared the NC and aMCI groups, widespread differences in $D_{\mathrm{e}, \perp}$ were evident, particularly in the corpus callosum (splenium, genu, and body), forceps major, and arcuate and uncinate fasciculi, while $D_{e, \|}$ was significantly different in periventricular regions, including the corpus callosum, anterior and posterior limbs of the internal capsule, and the uncinate and superior longitudinal fasciculi. In comparing aMCI and $\mathrm{AD}$ groups, differences in $D_{\mathrm{e}, \perp}$ were observed in more circumscribed WM tracts (eg, the splenium of the corpus callosum and optic radiations). In contrast, AWF was significantly different between the aMCI and AD groups in widespread lateral clusters, particularly in the corticocortical fibers, splenium of the corpus callosum, forceps major, anterior limb of the internal capsule, corona radiata, and cingulum. Accordingly, widespread differences were found between NC and patients with $\mathrm{AD}$ in $D_{\mathrm{e}, \perp}, D_{\mathrm{e}, \|}$, and AWF.

Given that the WMTI metrics $\left(D_{\mathrm{e}, \perp}, D_{\mathrm{e}, \|}\right.$, and AWF) were shown to be more sensitive (ie, detected more significantly different clusters) than the diffusivity/kurtosis metrics in distinguishing NC from patients with aMCI and subjects with aMCI from those with AD by using TBSS, ROI analyses of the corpus callosum were restricted to the 4 WMTI metrics. Table 2 lists the means and SDs of the 4 ROIs for each WMTI metric and the post hoc ANCOVA results. (On-line Table 1 provides these data for the standard diffusivity and kurtosis metrics.) The results in the ROI analyses for the WMTI metrics were consistent with the TBSS results that identified distinct WMTI metrics distinguishing NC from patients with aMCI and subjects with aMCI from those with $\mathrm{AD} . D_{\mathrm{e}, \|}$ was significantly increased in the aMCI group than in NC in all ROIs, while $D_{\mathrm{e}, \perp}$ was also significantly increased in the aMCI group than in NC in the genu. On the other hand, the AWF in the skeleton was significantly decreased in those with $\mathrm{AD}$ than in those with aMCI, while $D_{e, \perp}$ was significantly increased in the AD group than in the aMCI group in the splenium. Also similar to the TBSS results, the NC group was significantly different from the AD group in all ROIs and metrics except for $D_{\text {axon }}$.
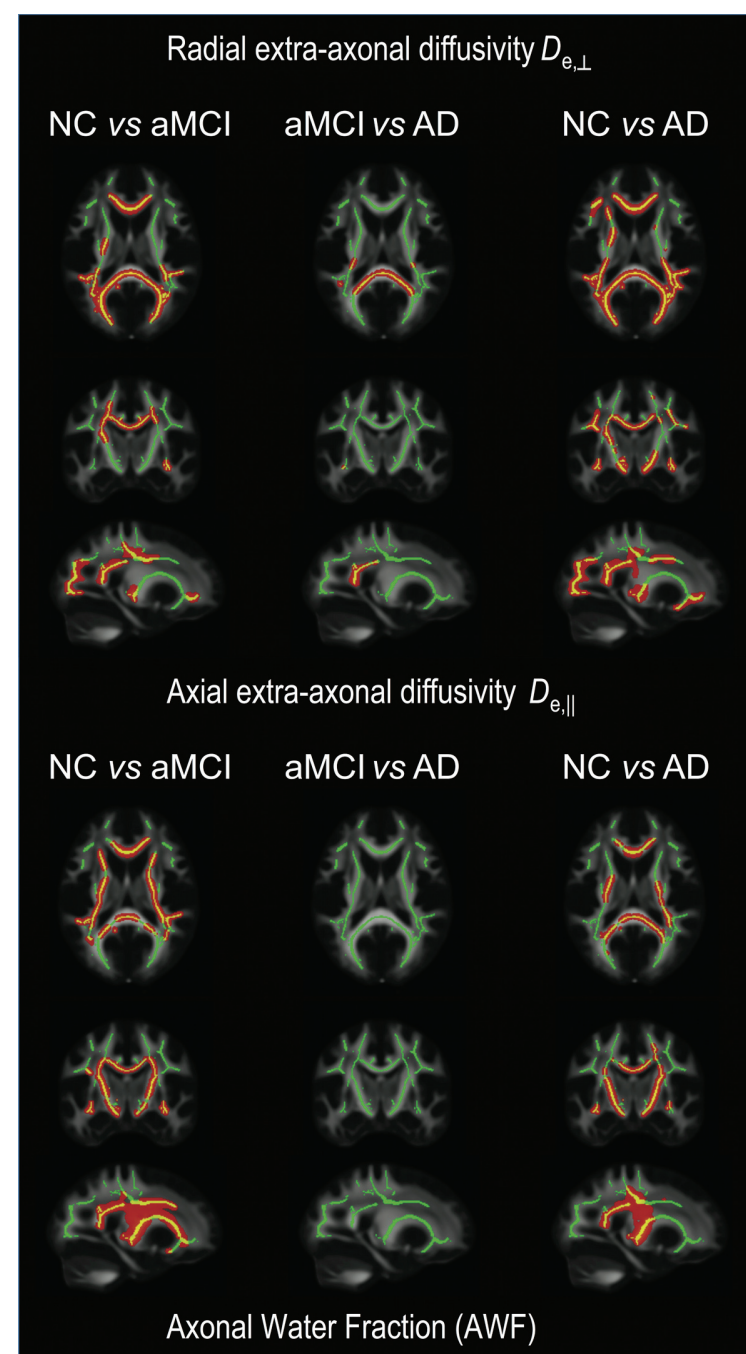

NC vs AD

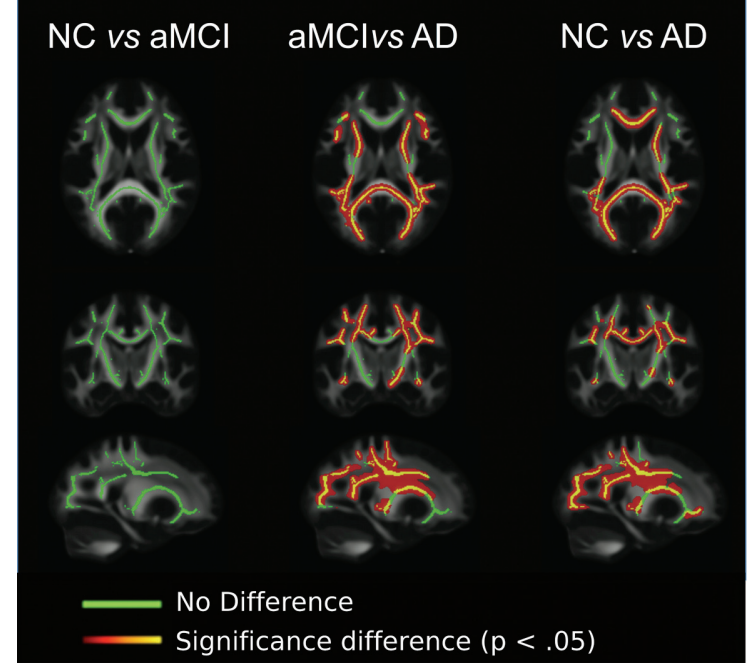

FIG 2. TBSS results showing all 3 group comparisons for WMTI metrics of radial extra-axonal diffusivity, axial extra-axonal diffusivity, and axonal water fraction: Clusters of significantly different voxels $(P<$ $.05)$ are shown in red-orange and overlaid on the FMRIB FA template, together with the mean skeleton (green). Clusters of increased radial extra-axonal diffusivity are found both for $\mathrm{NC}$ versus $\mathrm{MCl}$ groups and for $\mathrm{MCl}$ versus $\mathrm{AD}$ groups. Clusters of increased axial extra-axonal diffusivity are found for $\mathrm{NC}$ versus $\mathrm{MCl}$ groups, and clusters of decreased AWF are observed for $\mathrm{MCl}$ versus $\mathrm{AD}$ groups. 
Table 2: ANCOVA pos hoc and AUC results of the WMTI metrics of each corpus callosum ROI and the FA skeleton

\begin{tabular}{|c|c|c|c|c|c|c|c|c|c|c|}
\hline & \multirow[b]{2}{*}{ ROI } & \multirow{2}{*}{$\frac{\mathrm{NC}(n=15)}{\text { Mean } \pm \text { SD }}$} & \multirow{2}{*}{$\frac{\operatorname{aMCl}(n=12)}{\text { Mean } \pm \text { SD }}$} & \multirow{2}{*}{$\frac{A D(n=14)}{\text { Mean } \pm \text { SD }}$} & \multicolumn{2}{|c|}{$\mathrm{NC}$ vs aMCl } & \multicolumn{2}{|c|}{ aMCI vs $A D$} & \multicolumn{2}{|c|}{ NC vs $A D$} \\
\hline & & & & & $P$ Value & AUC & $P$ Value & AUC & $P$ Value & AUC \\
\hline \multirow[t]{4}{*}{ AWF } & Genu & $0.35 \pm 0.03$ & $0.33 \pm 0.03$ & $0.32 \pm 0.05$ & .25 & 0.75 & .43 & 0.67 & $.01^{\mathrm{a}, \mathrm{b}}$ & 0.78 \\
\hline & Body & $0.35 \pm 0.03$ & $0.33 \pm 0.03$ & $0.32 \pm 0.04$ & .33 & 0.71 & .35 & 0.65 & $.01^{\mathrm{a}, \mathrm{b}}$ & 0.76 \\
\hline & Splenium & $0.41 \pm 0.03$ & $0.39 \pm 0.03$ & $0.35 \pm 0.06$ & .36 & 0.69 & $.02^{\mathrm{a}}$ & 0.79 & $<.01^{c, b}$ & 0.88 \\
\hline & Skeleton & $0.39 \pm 0.02$ & $0.38 \pm 0.02$ & $0.36 \pm 0.04$ & .93 & 0.64 & $.01^{\mathrm{a}, \mathrm{b}}$ & 0.84 & $<.01^{\mathrm{d}, \mathrm{b}}$ & 0.83 \\
\hline \multirow[t]{4}{*}{$D_{\text {axon }}$} & Genu & $1.09 \pm 0.06$ & $1.09 \pm 0.05$ & $1.10 \pm 0.09$ & 1.00 & 0.66 & .88 & 0.53 & .88 & 0.53 \\
\hline & Body & $1.22 \pm 0.10$ & $1.18 \pm 0.07$ & $1.19 \pm 0.10$ & .65 & 0.64 & .98 & 0.54 & .75 & 0.56 \\
\hline & Splenium & $1.20 \pm 0.09$ & $1.17 \pm 0.05$ & $1.15 \pm 0.12$ & .82 & 0.63 & .62 & 0.71 & .25 & 0.71 \\
\hline & Skeleton & $0.96 \pm 0.05$ & $0.95 \pm 0.04$ & $0.93 \pm 0.07$ & 1.00 & 0.66 & .57 & 0.77 & .49 & 0.68 \\
\hline \multirow[t]{4}{*}{$D_{\mathrm{e}, \|}$} & Genu & $2.90 \pm 0.13$ & $3.06 \pm 0.07$ & $3.10 \pm 0.17$ & $.01^{\mathrm{a}, \mathrm{b}}$ & 0.86 & .57 & 0.60 & $<.01^{\mathrm{c}, \mathrm{b}}$ & 0.85 \\
\hline & Body & $2.83 \pm 0.11$ & $3.01 \pm 0.10$ & $2.99 \pm 0.15$ & $<.01^{\mathrm{d}, \mathrm{b}}$ & 0.89 & .93 & 0.54 & $<.01^{\mathrm{d}, \mathrm{b}}$ & 0.79 \\
\hline & Splenium & $2.79 \pm 0.11$ & $2.93 \pm 0.11$ & $2.95 \pm 0.11$ & $.01^{\mathrm{a}, \mathrm{b}}$ & 0.82 & .78 & 0.57 & $<.01^{\mathrm{d}, \mathrm{b}}$ & 0.88 \\
\hline & Skeleton & $2.30 \pm 0.07$ & $2.39 \pm 0.04$ & $2.40 \pm 0.07$ & $<.01^{\mathrm{c}, \mathrm{b}}$ & 0.95 & .88 & 0.53 & $<.01^{\mathrm{c}, \mathrm{b}}$ & 0.92 \\
\hline \multirow[t]{4}{*}{$D_{\mathrm{e}, \perp}$} & Genu & $1.33 \pm 0.11$ & $1.52 \pm 0.16$ & $1.58 \pm 0.27$ & $.01^{a, b}$ & 0.91 & .30 & 0.64 & $<.01^{\mathrm{c}, \mathrm{b}}$ & 0.91 \\
\hline & Body & $1.43 \pm 0.10$ & $1.58 \pm 0.12$ & $1.62 \pm 0.22$ & $.03^{\mathrm{a}}$ & 0.88 & .49 & 0.63 & $<.01^{\mathrm{c}, \mathrm{b}}$ & 0.80 \\
\hline & Splenium & $1.15 \pm 0.10$ & $1.29 \pm 0.12$ & $1.43 \pm 0.25$ & .06 & 0.87 & $<.01^{\mathrm{d}, \mathrm{b}}$ & 0.77 & $<.01^{\mathrm{c}, \mathrm{b}}$ & 0.93 \\
\hline & Skeleton & $1.06 \pm 0.07$ & $1.15 \pm 0.07$ & $1.20 \pm 0.16$ & $.04^{\mathrm{a}}$ & 0.89 & .06 & 0.77 & $<.0^{\mathrm{c}, \mathrm{b}}$ & 0.89 \\
\hline
\end{tabular}

Note:-AUC indicates area under the receiver operating characteristic curve.

a $P<.05$.

${ }^{\mathrm{b}} P$ values indicate that the difference remains significant after applying a Bonferroni correction for multiple ROI comparisons.

${ }^{c} P<.001$.

${ }^{\mathrm{d}} \mathrm{P}<.01$.

\section{WMTI Metrics Predict aMCI and AD with High Diagnostic} Accuracy

Given the corroborating TBSS and ROI results, area under the receiver operating characteristic curve analyses were conducted to demonstrate the diagnostic accuracy of the WMTI metrics (Table 2; see On-line Table 1 for these results for the standard diffusivity and kurtosis metrics). $D_{\mathrm{e}, \perp}$ and $D_{\mathrm{e}, \|}$ yielded the highest classification accuracies for discriminating NC from patients with aMCI (area under the receiver operating characteristic curve $=0.82-$ $0.95)$, while aMCI was best discriminated from AD by the AWF (area under the receiver operating characteristic curve $=0.84$ ). When we compared across the 4 ROIs, the FA skeleton consistently yielded the highest area under the receiver operating characteristic curve values.

\section{Processing Speed Strongly Correlates with WMTI Metrics in $\mathrm{aMCl}$}

Consistent with prior reports of significant correlations between processing speed and MR imaging-based estimates of WM integrity, ${ }^{31-33}$ the CPS score was strongly correlated with the WMTI metrics of diffusivity in the extra-axonal space: $D_{\mathrm{e}, \|}(r=-0.82$, $P<.001), D_{\mathrm{e}, \perp}(r=-0.80, P=.002)$, and AWF $(r=0.82, P<$ .001 ), in the callosal body in aMCI-that is, lower CPS scores were associated with higher extra-axonal diffusivities in the callosal body in aMCI but not in any other group. CPS correlated with the AWF in the callosal body in aMCI, wherein higher CPS scores were associated with a higher AWF.

\section{DISCUSSION}

In this study, we demonstrated that WMTI metrics provide unique information regarding the specific underlying mechanisms of WM alterations that occur in the course of AD. By using TBSS, we confirmed prior research that showed increased mean, radial, and axial diffusivity, as well as decreased FA, in WM tracts in subjects with aMCI and AD compared with NC. ${ }^{9,34}$ We also observed decreased mean and radial kurtosis values in AD com- pared with subjects with aMCI and NC. However, the specific novelty of this work is our application of the non-Gaussian diffusion information from DKI to a tissue-modeling technique that provides a more nuanced conceptualization of the microstructural changes occurring across stages of the disease. With high diagnostic accuracy, increased diffusivity in the extra-axonal space $\left(D_{\mathrm{e}, \|}, D_{\mathrm{e}, \perp}\right)$ in several WM tracts sensitively distinguished $\mathrm{NC}$ from patients with aMCI, while widespread decreased AWF differentiated aMCI from $\mathrm{AD}$ groups. Additionally, these metrics of WM integrity in the body of the corpus callosum were strongly correlated with processing speed in aMCI. These findings have the following potential implications for the course and spatial progression of WM degeneration in $\mathrm{AD}$, as well as the mechanisms by which these changes occur.

\section{Alterations in Extra-Axonal Diffusion as Biomarkers of the Earliest Stages of $A D$}

$\mathrm{AD}$ in its earliest stage is a new target phase for clinical trials because it is argued that addressing early pathologic changes may increase the likelihood of curtailing the disease. ${ }^{2}$ The significant difference in extra-axonal diffusivity both in the radial $\left(D_{\mathrm{e}, \perp}\right)$ and axial $\left(D_{\mathrm{e}, \|}\right)$ directions between $\mathrm{NC}$ and aMCI groups suggests that pathologic changes in the extra-axonal space, including demyelination, loss of oligodendrocytes, astrocytosis, or extracellular inflammation may be especially salient in the earliest stages of disease. Our data provide strong evidence that increased $D_{\mathrm{e}, \|}$ mainly occurs in periventricular regions in this stage, while $D_{\mathrm{e}, \perp}$ is increased in widespread lateral regions from NC through aMCI and into $\mathrm{AD}$. These observations suggest that $D_{\mathrm{e}, \perp}$ and $D_{\mathrm{e}, \|}$ may be sensitive to different underlying mechanisms, in which $D_{\mathrm{e}, \perp}$ may be more sensitive to myelin breakdown than $D_{\mathrm{e}, \| .}{ }^{18,27}$ Indeed, $D_{e, \perp}$ was significantly increased in patients with aMCI in the late-myelinating genu than in NC, and it was significantly increased in those with $\mathrm{AD}$ in the early-myelinating splenium and optic tracts than in patients with aMCI. This observed 
pattern appears consistent with the hypothesis of myelin breakdown being a significant pathogenic process in the course of $\mathrm{AD},{ }^{11}$ first observed in late-myelinating tracts early in the disease.

\section{Alterations in AWF as a Biomarker of AD Progression}

Apart from potential myelin changes, our study also provides evidence for decreased AWF, which has been recently proposed as a specific marker of axonal density loss. ${ }^{27}$ This finding corroborates known neurodegenerative processes in $\mathrm{AD}$ but further suggests that this occurs particularly in the transition from aMCI to AD. The significant decreases in AWF in the corticocortical fibers may suggest a reduction in axons due to Wallerian degeneration secondary to distal cortical atrophy as the primary pathologic event in these regions. ${ }^{35}$ However, we also observed increased $D_{\mathrm{e}, \perp}$ in the early-myelinating splenium of the corpus callosum from aMCI to $\mathrm{AD}$, suggesting that myelin and axonal changes co-occur in advanced disease.

Definitive conclusions regarding the temporality of these changes are precluded by the cross-sectional design of this study, but these findings certainly support previous research indicating that both myelin breakdown and Wallerian degeneration are implicated in $\mathrm{AD} .{ }^{34}$ Interestingly, we did not find any significant results for $D_{\text {axon }}$, a marker of intra-axonal injury and axonal beading. ${ }^{28}$ Although it is possible that the WM model is not sensitive to changes in the intra-axonal environment in $\mathrm{AD}$, these negative findings encourage further speculation on potential disease-related factors that preferentially impact axonal density rather than the intra-axonal environment.

\section{Correlation with Processing Speed}

Processing speed peaks at around the third and fourth decade of life, slowly declines with age, and facilitates other higher-order cognitive processes such as verbal memory retrieval. ${ }^{36,37} \mathrm{Al}-$ though the neurobiologic mechanisms of processing speed have yet to be fully determined, histologic and ethologic studies of brain aging demonstrate that myelin integrity supports the rapid transmission necessary for the distributed neural networks that facilitate cognitive functions. ${ }^{38-40}$ Human MR imaging studies provide further evidence for the association between estimates of myelin integrity in the anterior and posterior corpus callosum and processing speed in healthy older adults, ${ }^{31-33}$ but these associations are less examined in the course of $\mathrm{AD}$ and have not been tested by using the WMTI metrics modeled to estimate specific WM microstructure. In this study, the WMTI metrics $D_{\mathrm{e}, \perp}, D_{\mathrm{e}, \|}$, and AWF yielded strong correlations with processing speed in aMCI. Our results are unique in that these correlations were exclusive to the body of the corpus callosum, rather than the genu or splenium, which were the regions reported in prior studies. ${ }^{31-33}$ While these preliminary findings require replication before we can conjecture the functional relevance of the callosal body to cognition, they do provide unique insights into WM involvement in $\mathrm{AD}$ and to the links between diffusion MR imaging and behavioral metrics relevant to detecting disease onset and tracking its progression. For instance, it is possible that the strong correlations between the WMTI and processing speed metrics are due to the optimal level of variability in both metrics at the aMCI stage because of the presence of varying degrees of pathologic severity. This conceptualization suggests that changes in these metrics may signal a transition from normal aging to aMCI, though confirmation of this possibility is needed by using longitudinal data and other sensitive neurocognitive measures that have sufficient psychometric properties and are of clinical relevance to the progressive stages of disease.

\section{Clinical Implications and Future Directions}

This study demonstrates the sensitivity of WMTI metrics and their high diagnostic accuracy, which support the clinical potential of these metrics as biomarkers for the earliest stages of $\mathrm{AD}$, disease progression, or treatment response. In particular, we report that axial and radial extra-axonal diffusivities most accurately distinguish patients with aMCI from $\mathrm{NC}$, suggesting that these may be biomarkers for further exploration in the search for in vivo $M R$ imaging markers of the earliest brain changes in the course of $\mathrm{AD}$. On the other hand, our finding of decreased AWF from aMCI to $\mathrm{AD}$ could indicate that targeting axonal loss may be a possible strategy for slowing the trajectory of disease. Interestingly, while specific WMTI metrics most sensitively and accurately detect the differences between the $\mathrm{NC}$ and aMCI groups and between the aMCI and AD groups, we observed that the standard diffusivity metrics are the most sensitive in detecting differences between NC and patients with $\mathrm{AD}$ (Fig 1). This may, in part, be explained by the high specificity of the WMTI metrics to distinguish between groups, respectively. For example, $D_{\mathrm{e}, \|}$ is only significantly different between $\mathrm{NC}$ and patients with aMCI, while AWF is only significantly different between the aMCI and $\mathrm{AD}$ groups. As a result, diffusivity metrics may be overall more sensitive in detecting differences between NC and patients with $\mathrm{AD}$ as they are a combination of several WMTI metrics. However, our finding of changes in the extra-axonal diffusivity from NC to aMCI may be of keen interest to the field of $\mathrm{AD}$ at large, which is currently focused on developing biomarkers for early detection. Moreover, in aMCI, these metrics yielded strong correlations with processing speed, further providing their functional relevance to this disease. Thus, albeit requiring further replication and validation, the WMTI metrics may provide promising methods of detection and description of WM changes that occur in the course of AD.

Subsequent investigations of WMTI metrics may be improved by using multiple image processing and analytic methods. In this study, which used both TBSS and the conventional ROI analyses, the WMTI metric values over the FA skeleton, as derived from TBSS, yielded the highest area under the receiver operating characteristic curve values. Although the results of both methods were similar, TBSS may be the preferred approach because this standardized procedure minimizes preprocessing, obviates subjective ROI outlining, and naturally selects WM voxels consisting of strongly aligned fibers in which the WMTI metrics are valid and partial volume effects are suppressed. Validation of the WMTI metrics is also of crucial importance, and it would be particularly useful to evaluate the sensitivity and specificity of both $D_{\mathrm{e}, \perp}$ and $D_{\mathrm{e}, \|}$ to the different pathologic processes in the extra-axonal space (eg, gliosis, demyelination, inflammation) by using both histologic validation and other imaging modalities (eg, PET). 


\section{Limitations}

This study has some limitations that may be addressed in future research. First, this cross-sectional study only simulates the potential significance of the WMTI metrics in predicting conversion to aMCI or AD. Confirming these hypothesized changes by using a longitudinal design would be ideal. Second, WM lesion load may have influenced the diffusion metrics, though we did not find any significant differences in lesion load or vascular disease burden at the group level. While the extent of WM lesions associated with the metrics of WM integrity as defined via diffusion MR imaging remains under investigation, ${ }^{41}$ improvement to this work can include both clinician-rated and automated methods of quantifying lesion burden or other vascular compromise. Third, because the underlying WM model is only applicable in regions consisting of aligned fiber bundles, we limited our analysis to voxels with high FA. Last, the sample size of this preliminary study limits the generalizability of these results and the applicability of the diagnostic accuracy of these metrics to community-based samples in which aMCI and AD are less prevalent.

\section{CONCLUSIONS}

This is the first study to demonstrate that WM tract integrity metrics significantly differentiate healthy controls and subjects with amnestic MCI and AD, yield diagnostically sensitive information regarding the underlying mechanisms of WM degeneration, and correlate with processing speed, a cognitive function most relevant to WM integrity. These novel WMTI metrics yield insights into underlying disease mechanisms in $\mathrm{AD}$, which, with further investigation, can potentially yield promising biomarkers of the earliest brain changes in $\mathrm{AD}$.

\section{ACKNOWLEDGMENTS}

We thank Vitria Adisetiyo, José Raya, and Henry Rusinek for technical assistance and helpful discussions while preparing this manuscript. We also thank Amanda Allen and Jane Kwon for their assistance with data analysis and research coordination.

Disclosures: Els Fieremans—RELATED: Grant: Alzheimer Drug Discovery Foundation, * Litwin Foundation, ${ }^{\star}$ Comments: This study was outlined as part of a research grant from the Alzheimer Drug Discovery Foundation (principal investigator [PI]: E. Fieremans), active between June 1, 2011 and May 31, 2012. UNRELATED: Siemens Medical owns a royalty-free nonexclusive license for diffusional kurtosis imaging with the pending patent held by New York University. I am one of the inventors. Jens $\mathrm{H}$. Jensen—RELATED: Grant: NIH (R01AG027852), ${ }^{*}$ Alzheimer Drug Discovery Foundation, ${ }^{\star}$ Litwin Foundation, ${ }^{\star}$ Comments: This article includes work that was funded in part by NIH grant R01AG027852 (PI:J.A. Helpern). Additional funding was provided by the Alzheimer Drug Discovery Foundation (PI: E. Fieremans) and the Litwin Foundation (PI: J.A. Helpern), UNRELATED: Siemens Medical owns a royalty-free nonexclusive license for diffusional kurtosis imaging with the pending patent held by New York University. I am one of the inventors. Maria F. Falangola—RELATED: Grant: NIH (R01AG027852), ${ }^{*}$ Alzheimer Drug Discovery Foundation, " ${ }^{*}$ Litwin Foundation. ${ }^{*}$ Ali Tabesh—UNRELATED: Siemens Medical owns a royalty-free nonexclusive license for diffusional kurtosis imaging with the pending patent held by New York University. I am one of the inventors. M. Vittoria Spampinato-RELATED: Grant: NIH (R01AG027852). * Dmitry S. Novikov—RELATED: Grant: Litwin Foundation. * Steven H. Ferris—RELATED: Grant: National Institute on Aging Alzheimer's Disease Core Center Grant, Comments: supported clinical/diagnostic evaluations for the clinical assessments of study participants prior to their initial enrollment in the current study.

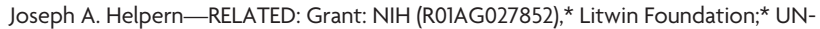
RELATED: Siemens Medical owns a royalty-free nonexclusive license for diffusional kurtosis imaging with the pending patent held by New York University. I am one of the inventors. * Money paid to the institution.

\section{REFERENCES}

1. Morris JC. Mild cognitive impairment and preclinical Alzheimer's disease. Geriatrics 2005; (suppl):9-14

2. Reiman EM, Langbaum JB, Tariot PN. Alzheimer's prevention initiative: a proposal to evaluate presymptomatic treatments as quickly as possible. Biomark Med 2010;4:3-14

3. Duyckaerts C, Delatour B, Potier MC. Classification and basic pathology of Alzheimer disease. Acta Neuropathol 2009;118:5-36

4. Sjöbeck M, Haglund M, Englund E. Decreasing myelin density reflected increasing white matter pathology in Alzheimer's disease: a neuropathological study. Int J Geriatr Psychiatry 2005;20:919-26

5. Brun A, Englund E. A white matter disorder in dementia of the Alzheimer type: a pathoanatomical study. Ann Neurol 1986;19: 253-62

6. Englund E. Neuropathology of white matter changes in Alzheimer's disease and vascular dementia. Dement Geriatr Cogn Disord 1998; 9(suppl 1):6-12

7. Gouw AA, Seewann A, Vrenken H, et al. Heterogeneity of white matter hyperintensities in Alzheimer's disease: post-mortem quantitative MRI and neuropathology. Brain 2008;131(pt 12): 3286-98

8. Sjöbeck M, Haglund M, Englund E. White matter mapping in Alzheimer's disease: a neuropathological study. Neurobiol Aging 2006;27:673-80

9. Clerx L, Visser PJ, Verhey F, et al. New MRI markers for Alzheimer's disease: a meta-analysis of diffusion tensor imaging and a comparison with medial temporal lobe measurements. J Alzheimers Dis 2012;29:405-29

10. Di Paola M, Spalletta G, Caltagirone C. In vivo structural neuroanatomy of corpus callosum in Alzheimer's disease and mild cognitive impairment using different MRI techniques: a review. J Alzheimers Dis 2010;20:67-95

11. Bartzokis G. Alzheimer's disease as homeostatic responses to agerelated myelin breakdown. Neurobiol Aging 2011;32:1341-71

12. Englund E, Brun A. White matter changes in dementia of Alzheimer's type: the difference in vulnerability between cell compartments. Histopathology 1990;16:433-39

13. Coleman M. Axon degeneration mechanisms: commonality amid diversity. Nat Rev Neurosci 2005;6:889-98

14. Basser PJ, Pierpaoli C. Microstructural and physiological features of tissues elucidated by quantitative-diffusion-tensor MRI. J Magn Reson B 1996;111:209-19

15. Jensen JH, Helpern JA. MRI quantification of non-Gaussian water diffusion by kurtosis analysis. NMR Biomed 2010;23:698-710

16. Jensen JH, Helpern JA, Ramani A, et al. Diffusional kurtosis imaging: the quantification of non-gaussian water diffusion by means of magnetic resonance imaging. Magn Reson Med 2005;53: 1432-40

17. Helpern JA, Falangola MF, Tabesh A, et al. Diffusional kurtosis imaging in mild cognitive impairment and Alzheimer's disease. In: Proceedings of the 19th Annual Meeting of the International Society for Magnetic Resonance in Medicine, Montreal, Canada. May 6-13, 2011:4217

18. Fieremans E, Jensen JH, Helpern JA. White matter characterization with diffusional kurtosis imaging. Neuroimage 2011;58:177-88

19. Reisberg B, Ferris SH, De Leon MJ, et al. The Global Deterioration Scale for assessment of primary degenerative dementia. Am J Psychiatry 1982;139:1136-39

20. Wechsler D. Manual for the Wechsler Memory Scale. 3rd ed. San Antonio, Texas: Psychological Corporation; 1997

21. American Psychiatric Association. Diagnostic and Statistical Manual of Mental Disorders. 4th ed. Washington, DC: American Psychiatric Association

22. McKhann G, Drachman D, Folstein M, et al. Clinical diagnosis of Alzheimer's disease: report of the NINCDS-ADRDA Work Group under the auspices of Department of Health and Human Services Task Force on Alzheimer's Disease. Neurology 1984;34:939-44 
23. Folstein MF, Folstein SE, McHugh PR. "Mini-mental state": a practical method for grading the cognitive state of patients for the clinician. J Psychiatr Res 1975;12:189-98

24. Weintraub S, Salmon D, Mercaldo N, et al. The Alzheimer's Disease Centers' Uniform Data Set (UDS): the neuropsychologic test battery. Alzheimer Dis Assoc Disord 2009;23:91-101

25. Shirk SD, Mitchell MB, Shaughnessy LW, et al. A web-based normative calculator for the uniform data set (UDS) neuropsychological test battery. Alzheimers Res Ther 2011;3:32

26. Fieremans E, Novikov DS, Jensen JH, et al. Monte Carlo study of a two-compartment exchange model of diffusion. NMR Biomed 2010;23:711-24

27. Fieremans E, Jensen JH, Helpern JA, et al. Diffusion distinguishes between axonal loss and demyelination in brain white matter. In: Proceedings of the 20th Annual Meeting of the International Society for Magnetic Resonance Medicine, Melbourne, Australia; May 5-11, 2012

28. Hui ES, Fieremans E, Jensen JH, et al. Stroke assessment with diffusional kurtosis imaging. Stroke 2012;43:2968-73

29. Smith SM, Jenkinson M, Johansen-Berg H, et al. Tract-based spatial statistics: voxelwise analysis of multi-subject diffusion data. Neuroimage 2006;31:1487-505

30. Smith SM, Johansen-Berg H, Jenkinson M, et al. Acquisition and voxelwise analysis of multi-subject diffusion data with tract-based spatial statistics. Nat Protoc 2007;2:499-503

31. Kochunov P, Coyle T, Lancaster J, et al. Processing speed is correlated with cerebral health markers in the frontal lobes as quantified by neuroimaging. Neuroimage 2010;49:1190-99

32. Kennedy KM, Raz N. Aging white matter and cognition: differ- ential effects of regional variations in diffusion properties on memory, executive functions, and speed. Neuropsychologia 2009; 47:916-27

33. Lu PH, Lee GJ, Raven EP, et al. Age-related slowing in cognitive processing speed is associated with myelin integrity in a very healthy elderly sample. J Clin Exp Neuropsychol 2011;33: 1059-68

34. Sexton CE, Kalu UG, Filippini N, et al. A meta-analysis of diffusion tensor imaging in mild cognitive impairment and Alzheimer's disease. Neurobiol Aging 2011;32:2322.e5-18

35. Fellgiebel A, Schermuly I, Gerhard A, et al. Functional relevant loss of long association fibre tracts integrity in early Alzheimer's disease. Neuropsychologia 2008;46:1698-706

36. Hedden T, Lautenschlager G, Park DC. Contributions of processing ability and knowledge to verbal memory tasks across the adult lifespan. Q J Exp Psychol A 2005;58:169-90

37. Salthouse TA. Aging and measures of processing speed. Biol Psychol 2000;54:35-54

38. Meyer A. Paul Flechsig's system of myelogenetic cortical localization in the light of recent research in neuroanatomy and neurophysiology: part I. Can J Neurol Sci 1981;8:1-6

39. Peters A, Rosene DL, Moss MB, et al. Neurobiological bases of agerelated cognitive decline in the rhesus monkey. J Neuropathol Exp Neurol 1996;55:861-74

40. Yakovlev P, Lecours A. Regional Development of the Brain in Early Life. Boston: Blackwell Scientific Publications; 1967

41. Maillard P, Carmichael O, Harvey D, et al. FLAIR and diffusion MRI signals are independent predictors of white matter hyperintensities. AJNR Am J Neuroradiol 2013;34:54-61 